\title{
Robot Simulation for Control Design
}

\author{
Leon Žlajpah \\ Jožef Stefan Institute \\ Slovenia
}

\begin{abstract}
Research in the field of robotics is tightly connected to simulation tools for many reasons. On one side, simulation supports the development of new advanced control algorithms and on the other side, it is always not feasible to build a whole robot system to test some algorithms or it is not safe to perform tests on a real system (at least in the first design stages). The simulation has also a very important role for off-line programming, to design mechanical structure of robots, to design robotic cells and production lines, etc.

In the paper, an overview of the simulation in robotics is given and some topics like: how simulation makes things easier, advantages and backdraws of the simulation in robotics, virtual and real world, are pointed out. The scope of the paper is the role of the simulation in different fields of robotics, especially the dynamic simulation of robot manipulators. We present an integrated environment for the design and testing of advanced robot control schemes. The main capabilities of such environment are: the simulation of the kinematics and dynamics of manipulators, the integration of different sensor systems like vision and force sensors, scenarios for complex robot tasks, the visualization of robots and their environment and the integration of real robots in the simulation loop. We give an overview of simulation and visualization tools suitable for the simulation of robot systems using general dynamic engines and graphic languages. Finally, we present some typical simulation examples in different fields of robotics from offline programming, mobile robots to space robotics.
\end{abstract}

\section{Introduction}

Simulation has been recognized as an important research tool since the beginning of the 20th century. In the beginning, simulation was first of all an academic research tool. The "good times" for simulation started with the development of computers. First, the analog computers and later the digital computers have boosted simulation to new levels. So, the simulation is now a powerful tool supporting the design, planning, analysis, and decisions in different areas of research and development. Simulation has become a strategic tool in many fields, used by many researchers, developers and by many manufacturers. Of course, robotics as a modern technological branch is no exception. Actually, in robotics simulation plays a very important role, perhaps more important than in many other fields and we like to present in the following some insight in the robotics from the simulation point of view.

\subsection{The role of simulation}

Being able to simulate opens a wide range of options for solving many problems creatively. You can investigate, design, visualize, and test an object or even if it does not exists. You can 
see the results of a system yet to be built. It is possible that your solutions may fail or even blow up, but only in simulation. So, using the simulation tools one can avoid injuries and damages, unnecessary changes in design after the production of parts has already started, to long cycle times in manufacturing process, and even unnecessary paper work. Simulation enables us to work even in four dimensions. For example, one can observe within a few minutes how a planned production will be realized in next month, or a fast process can be slowed down to observe all details in "slow motion". All these make things easier and cheaper. One of the problems in classical design and planning are "what-if" questions. Due to the system complexity many of them are often unasked or not answered. With up-to-date simulation tools one can deal with exact geometry, consider the dynamic characteristics of a system, include the man-machine interfaces, and visualize the object in 3D in detail. Having all these in mind there is no reason for avoiding any "what-if" question. The boundaries for what is possible or not are pushed far away especially in advanced virtual reality tools. Using simulator researchers may build experimental environments according to their own imagination. Complexity, reality, specificity can be gradually increased to a level where virtual systems can head to real challenges of the physical world and even beyond.

Simulation is a highly interdisciplinary field since it is widely used in all fields of research from engineering and computer science to economics and social science, and at different levels from academic research to manufactures. Of course, simulation has been also recognized as an important tool in robotics: in designing new products, investigating its performances and in designing applications of these products. Simulation allows us to study the structure, characteristics and the function of a robot system at different levels of details each posing different requirements for the simulation tools. As the complexity of the system under investigation increases the role of the simulation becomes more and more important.

\section{Simulation of robot manipulators}

The ways and methods in robotics research and development have always been influenced by the tools used. This is especially true when one considers the profound impact of recent technologies on robotics, especially the development of computers which have become indispensable when designing the complex systems like robots. Not many years ago, computing cost was still a significant factor to consider when deriving algorithms and new modeling techniques (Fenton \& Xi, 1994; Latombe, 1995; Zhang \& Paul, 1988). Nowadays, distributed computing, network technology and the computing power developed by commercial equipment open new possibilities for doing systems design and implementation. However, in spite of all that, the creativity of a human designer can not be left out in the design process. The best solution seems to be to provide the designer with proper tools which significantly increase his efficiency. Among them, the simulation has been recognized as an important tool in designing the new products, investigating their performances and also in designing applications of these products. For complex systems as robots, the simulation tools can certainly enhance the design, development, and even the operation of the robotic systems. Augmenting the simulation with visualization tools and interfaces, one can simulate the operation of the robotic systems in a very realistic way.

A large amount of simulation software is available for robot systems, and it is already being used extensively. The majority of the robot simulation tools focus on the motion of the robotic manipulator in different environments. As the motion simulation has a central role in all simulation systems they all include the kinematic or dynamic models of robot manipulators. Which type of models will be used depends on the objective of the simulation system. For 
example, trajectory planning algorithms rely on kinematic models. Similarly, the construction of a robotized cell can be simulated efficiently by using only kinematic models of robot manipulators, without considering the dynamics or drives. On the other hand, dynamic models are needed to design the actuators. For example, modern control systems of robotic manipulators use internally different robot kinematic and dynamic models to improve the performance.

To model and simulate a robot manipulator different approaches are possible. They can differ in the way the user builds the model. Block diagram oriented simulation software requires that the user describes the system by combining the blocks, and there are other packages requiring the manual coding. To overcome the problems which arise when the system is very complex (and the robots usually are) several approaches exist to automatically generate the kinematic and/or dynamic models of robots.

The simulation tools for robotic systems can be divided into two major groups: the tools based on general simulation systems and special tools for robot systems. The tools based on general simulation systems are usually special modules, libraries or user interfaces which simplify the building of robot systems and environments within these general simulation systems. One of the advantages of such integrated toolboxes is that they enable you to use other tools available in the simulation system to perform different tasks. For example, to design control system, to analyse simulation results, to visualize results, etc. There exist several general simulation tools which are used for simulation of robot systems like MATLAB/Simulink, Dymola/Modelica, 20-sim, Mathematica, etc. Special simulation tools for robots cover one or more tasks in robotics like off-line programming, design of robot work cells, kinematic and dynamic analysis, mechanical design. They can be specialized for special types of robots like mobile robots, underwater robots, parallel mechanisms, or they are assigned to predefined robot family.

Simulation tools for robotic systems differ from each other regarding the aspect of the robot research they support, how open they are or on which platforms they work. However, many tools are not always fulfilling all the requirements of the research activities in robotic laboratories like reconfigurability, openness and ease of use, etc.

Reconfigurability and openness are features already recognized by many as essential in the development of advanced robot control algorithms (Alotto et al., 2004; Lambert et al., 2001; Lippiello et al., 2007). Not only is it important to have easy access to the system at all levels (e.g. from high-level supervisory control all the way down to fast servo loops at the lowest level), but it is a necessity to have open control architectures where software modules can be modified and exteroceptive sensors like force/torque sensors and vision systems can be easily integrated. Reconfigurability should also be reflected when more fundamental changes to the controller architecture are required, in the necessity of quickly being able to make modifications in the original design and verify the effect of these modifications on the system. In other words, the user should be able to quickly modify the structure of the control without having to alter the simulation system itself.

In the last decade the software has become more and more easy to use. This is still one of the main major issues when selecting a software tool. First of all, the tools are used by many users in a laboratory and not all of them have the same expertise. To boost the knowledge exchange, it is of benefit that they work with the same tools. Next, testing of different control algorithms on real robotic systems is in general not very user friendly: the algorithms usually have to be rewritten for the real-time execution and the different implementation details have to be considered (Lambert et al., 2001; Žlajpah, 2001). This forces the user to devote a large part of the design time to topics not connected with the main issues of the control de- 
sign, especially when he is not interested in software implementation issues. The ease of use becomes even more important when students are working with robots. In most cases they work in a laboratory for a shorter period, they are focused on their projects and they could become frustrated if they have to learn a lot of things not directly connected to their tasks. Finally, in research laboratories different robot systems are used equipped with more or less open proprietary hardware and software architecture. Therefore, it is much desired that the control design environment is unified, i.e. the same tools can be used for all robot systems.

The simulation tools for robotic systems can be divided into two major groups: tools based on general simulation systems and special tools for robot systems. Tools based on general simulation systems are usually represented as special modules, libraries or user interfaces which simplify the building of robot systems and environments within these general simulation systems (e.g. SolidWorks (RobotWorks, 2008)). On the other hand, special simulation tools for robots cover one or more tasks in robotics like off-line programming and design of robot work cells (e.g. Robcad (RobCAD, 1988)) or kinematic and dynamic analysis (Corke, 1996; SimMechanics, 2005). They can be specialized for special types of robots like mobile robots, underwater robots, parallel mechanisms, or they are assigned to predefined robot family. Depending on the particular application different structural attributes and functional parameters have to be modelled.

For the use in research laboratories, robot simulation tools focused on the motion of the robotic manipulator in different environments are important, especially those for the design of robot control systems (Corke, 1996; MSRS, 2008; SimMechanics, 2005; Webots, 2005). Recently, Microsoft Robotics Studio (MSRS, 2008) has been launched with a general aim to unify robot programming for hobbyist, academic and commercial developers and to create robot applications for a variety of hardware platforms. The system enables both remotely connected and robot-based scenarios using. NET and XML protocols. The simulation engine enables realtime physics simulation and interaction between simulated entities. Each part of the control loop can be substituted with the real or simulated hardware. Although the system is still under development, it is not easy to add new entity, for example a new robot or a new sensor. One of the major drawbacks seems to be the low data throughput rate, which does not allow the realization of complex control laws at high sampling frequency. Therefore, it is not clear yet if MSRS is appropriate for research robotics, especially for complex systems. Real time requirements are better solved in another programming/simulation framework, MCA2 (MCA2, 2008). MCA is a modular, network transparent and realtime capable $C / C++$ framework for controlling robots and other hardware. The main platform is Linux/RTLinux, but the support for Win32 and MCA OS/X also exists. However, it is still a complex system and therefore less appropriate for education and students projects.

\subsection{MATLAB based tools}

MATLAB is definitely one of the most used platforms for the modelling and simulation of various kind of systems and it is not surprising that it has been used intensively for the simulation of robotics systems. Among others the main reasons for that are its capabilities of solving problems with matrix formulations and easy extensibility. As an extension to MATLAB, SIMULINK adds many features for easier simulation of dynamic systems, e.q. graphical model and the possibility to simulate in real-time. Among special toolboxes that have been developed for MATLAB we have selected four: (a)Planar Manipulators Toolbox (Žlajpah, 1997), (b)Planar Manipulators Toolbox with SD/FAST (SD/FAST, 1994), (c)“A Robotic 


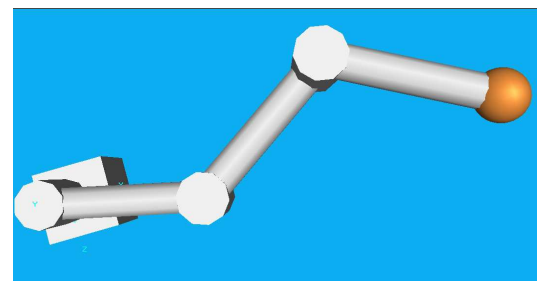

Fig. 1. Simple 3-R planar manipulator

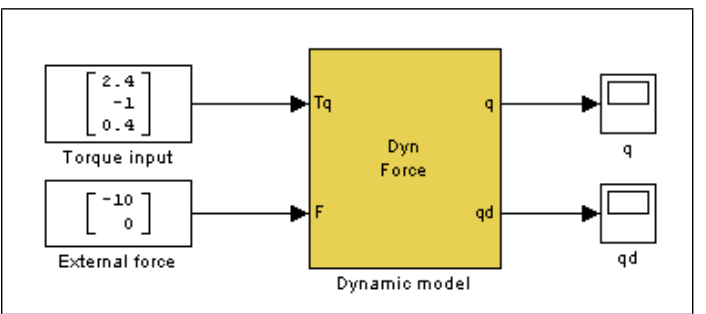

Fig. 2. Top level block scheme

Toolbox" (Corke, 1996), (d) "SimMechanics Toolbox" (SimMechanics, 2005) and (e) "20-sim" (Kleijn, 2009).

To illustrate different approaches to the dynamic simulation of robot manipulators we have selected as an object a simple planar manipulator which has 3 revolute joints acting in a plane as shown on Fig. 1. The main part of any simulation is the dynamic model. To focus on it, we simulate only the dynamics, without any task controller.

Let the configuration of the manipulator be represented by the vector $\boldsymbol{q}$ of $n$ joint positions, and the end-effector position (and orientation) by $m$-dimensional vector $\boldsymbol{x}$ of task positions. The joint and task coordinates are related by the following expressions

$$
\boldsymbol{x}=\mathbf{p}(\boldsymbol{q}), \quad \dot{\boldsymbol{x}}=\mathrm{J}(\boldsymbol{q}) \dot{\boldsymbol{q}}, \quad \ddot{\boldsymbol{x}}=\mathrm{J} \ddot{\boldsymbol{q}}+\dot{\mathrm{J}} \dot{\boldsymbol{q}}
$$

where $\mathbf{J}$ is the Jacobian matrix, and the overall dynamic behaviour of the manipulator is described by the following equation

$$
\boldsymbol{\tau}=\mathbf{H}(\boldsymbol{q}) \ddot{\boldsymbol{q}}+\mathbf{h}(\dot{\boldsymbol{q}}, \boldsymbol{q})+\boldsymbol{g}(\boldsymbol{q})-\boldsymbol{\tau}_{\mathrm{F}}
$$

where $\tau$ is the vector of control torques, $\mathbf{H}$ is the symmetric positive-definite inertia matrix, $\mathbf{h}$ is the vector of Coriolis and centrifugal forces, $\boldsymbol{g}$ is the vector of gravity forces, and vector $\boldsymbol{\tau}_{\mathrm{F}}$ represents the torques due to the external forces acting on the manipulator.

Fig. 2 shows the top level block scheme of the system. This scheme is the same in all cases, only the Dynamic model block is changed.

\section{(a) Planar Manipulators Toolbox}

Planar Manipulators Toolbox is intended for the simulation of planar manipulators with revolute joints and is based on Lagrangian formulation. Planar Manipulators Toolbox can be used to study kinematics and dynamics, to design control algorithms, for trajectory planning. It enables also real time simulation. Due to its concept it is a very good tool for education. To gain the transparency, special blocks have been developed to calculate the kinematic and dynamic models. These blocks are then used to build the desired model. Fig. 3 shows the dynamic model where an external force acts on the end-effector. The block dymodall which calculates the system vectors and matrices $\boldsymbol{x}, \mathbf{J}, \mathbf{J}, \mathbf{H}, \boldsymbol{h}$ and $\boldsymbol{g}$ and then joint accelerations are calculated using Lagrangian equation.

\section{(b) Planar Manipulators Toolbox with SD/FAST}

In this case we use Planar Manipulators Toolbox but the dynamic model is calculated SD/FAST library. SD/FAST can be used to perform analysis and design studies on any mechanical system which can be modelled as a set of rigid bodies interconnected by joints, influenced by forces, driven by prescribed motions, and restricted by constraints (SD/FAST, 1994). The dynamic model has the same structure as given in Fig. 3 except that the block dymodall 


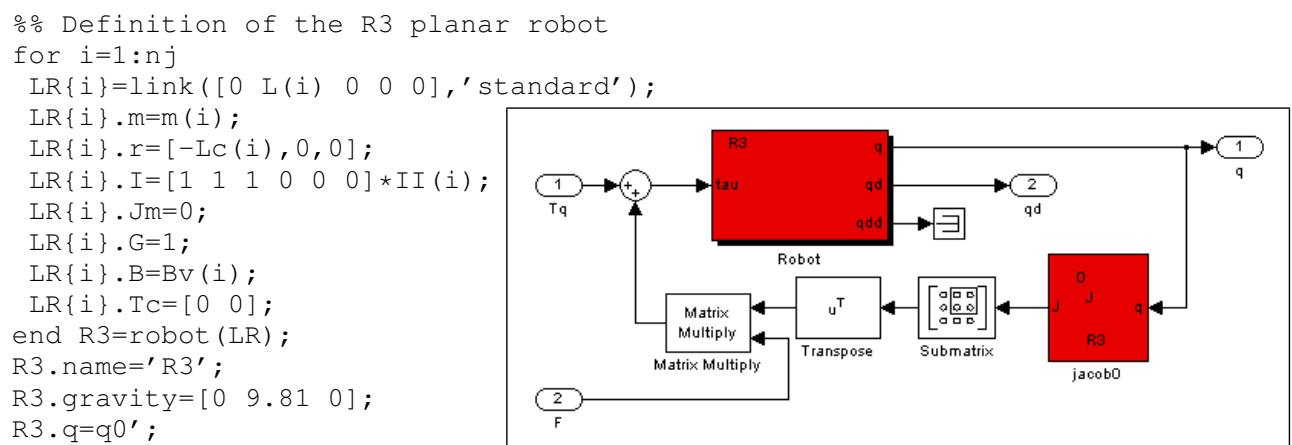

Fig. 5. Dynamic model (Robotics toolbox)

and coordinate systems, and to initiate and measure body motions (SimMechanics, 2005). To get a dynamic model of a robot manipulator we have first to build the link model, i.e. to connect link masses with joints as it is shown on Fig. 7. All link models are then connected together to the complete model (Fig. 6).

\section{(e) 20-sim}

Although 20-sim is a stand-alone simulation system (described later), it has a possibility to export the model to Simulink blocks as C-mex function. For comparison, we have modelled our robot manipulator using the 3D Mechanic Editor where you can model mechanical systems by specifying bodies, joints, sensors and actuators (Kleijn, 2009). To get a dynamic model of a robot manipulator we have first defined the links and then we have connected links with joints as it is shown on Fig. 8. Adding the trajectories generator, controllers and power amplifiers with gears a complete model of the system can be built (Fig. 9). Using the C code generator in 20-sim we have generated a Simulink block of the manipulator subsystem (R3). This block is then used in Simulink simulation scheme as shown in Fig. 2.

In all five cases it has been very easy to build the robot system. One of the differences between these tools is that special toolboxes for robot modelling have predefined more specific functions and blocks as the general toolboxes. The other difference is the execution time. In Fig. 10 we give the calculation time for the dynamic model for all five approaches. First we can see that SD/FAST is significantly faster than other and is increasing more slowly versus the

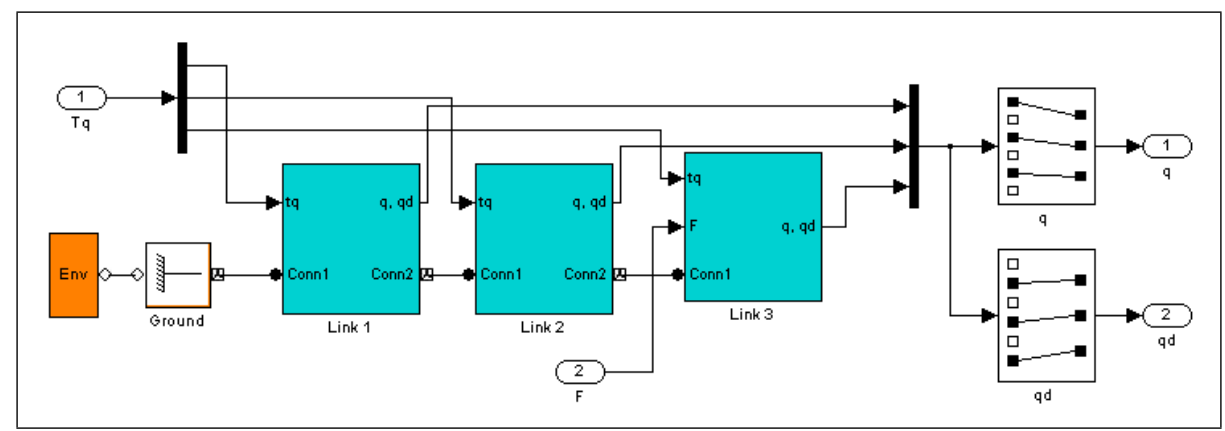

Fig. 6. Dynamic model of 3R manipulator (SimMechanics toolbox) 


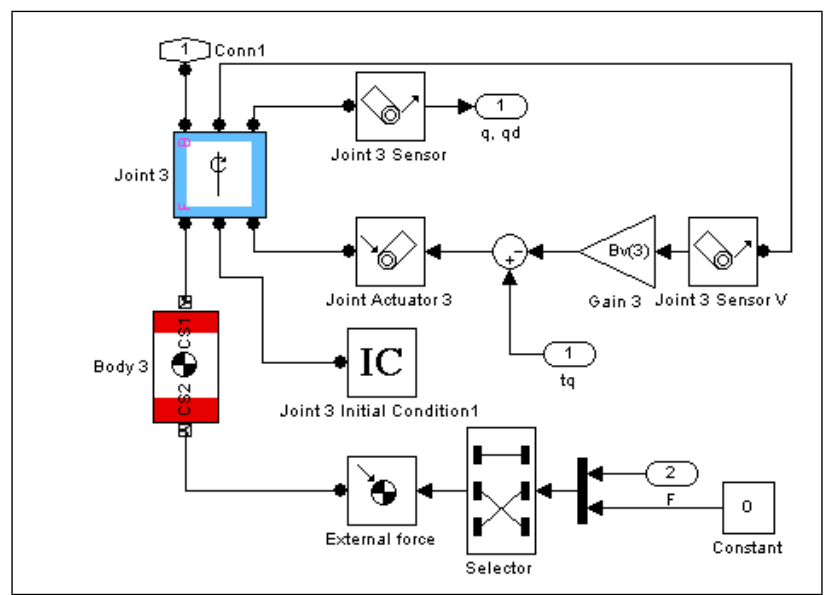

Fig. 7. Model of one link (SimMechanics toolbox)

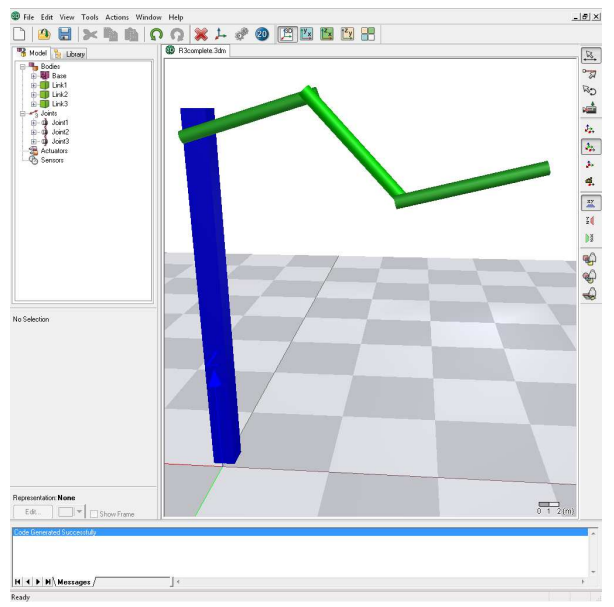

Fig. 8. Modelling robot manipulator using 20-sim 3D Mechanic Editor 


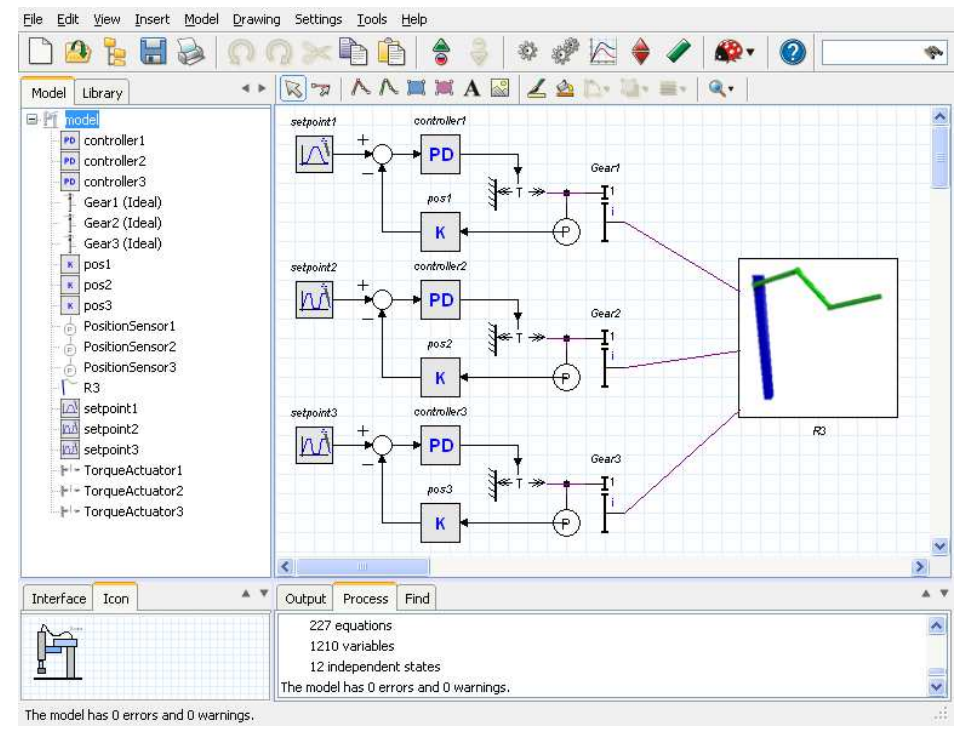

Fig. 9. Complete model of 3R manipulator (20-sim)

degrees-of freedom than other. Next, Planar Manipulators Toolbox is fast for small number of degrees-of-freedom and the execution time increases fast with the number of degrees-offreedom. The Robotics Toolbox is relatively fast as long as we use only the inverse dynamics (Note that in Fig. 10 only the calculation time for the inverse dynamic model is shown). Otherwise, e.g. for the calculation of the Jacobian matrix, it is significantly slower, because the calculation is based on M-functions. Also, the model generated in 20-sim is fast (simulation within 20-sim environment is even faster). A little slower is the SimMechanics Toolbox. In both cases the execution time versus the number of degrees-of-freedom increases similarly. However, if the models the of robot manipulators should be used in the controller (e.g. the Jacobian matrix), then SimMechanics Toolbox and 20-sim are not appropriate.

\subsection{Other general simulation systems}

Similarly as in MATLAB the robot system can be simulated in Dymola and Modelica, or 20sim. Here, the MultiBody library provides 3-dimensional mechanical components to model rigid multibody systems, such as robots. The robot system is built by connecting the blocks representing parts of the robot like link bodies, joints, actuators, etc. Fig. 11 shows the block scheme of a complete model of the KUKA robot including actuators, gears and the controller (Kazi \& Merk, 2002). Fig. 12 shows the simulation of a parallel robot manipulator with 20-sim (3D Mechanics Toolbox) (Kleijn, 2009).

Robotica is a computer aided design package for robotic manipulators based on Mathematica (Nethery \& Spong, 1994). It encapsulates many functions into a Mathematica package allowing efficient symbolic and numeric calculation of kinematic and dynamic equations for multi-degree-of-freedom manipulators. Robotica is intended, first of all, for model generation and analysis of robotic systems and for simulation. 


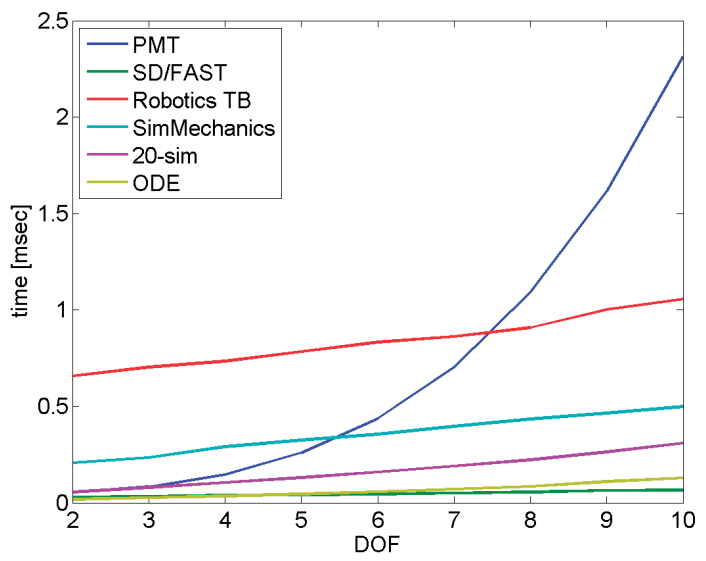

Fig. 10. Comparison of the calculation time versus number of DOF for the dynamic model of $n$-R planar robot manipulator

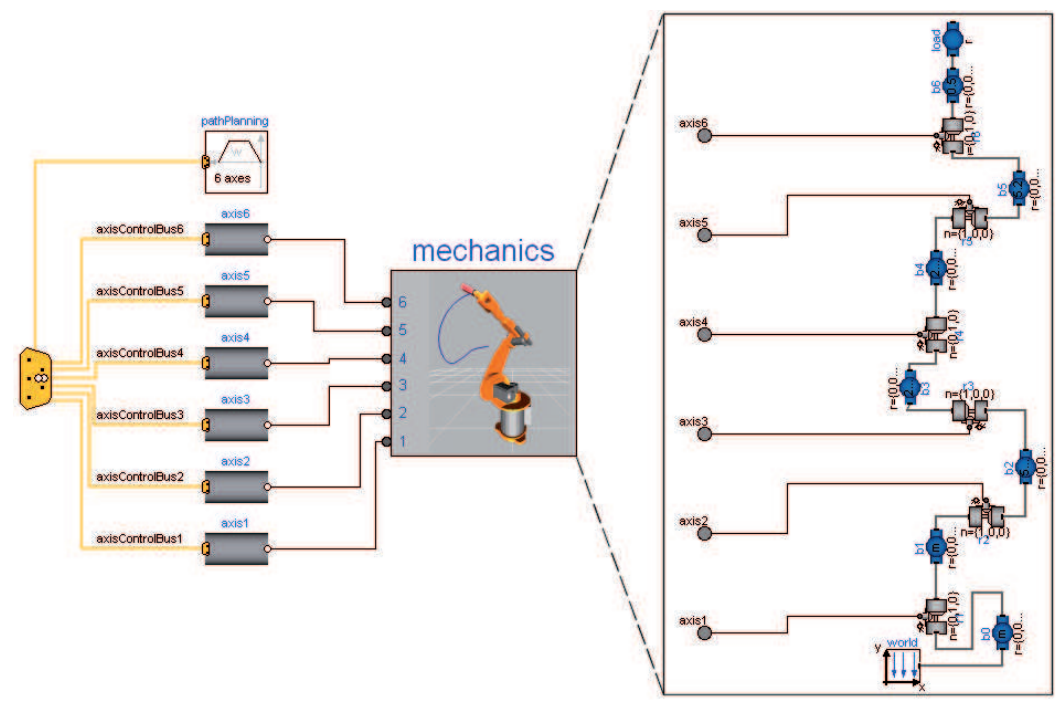

Fig. 11. Simulation of a robot with Modelica 


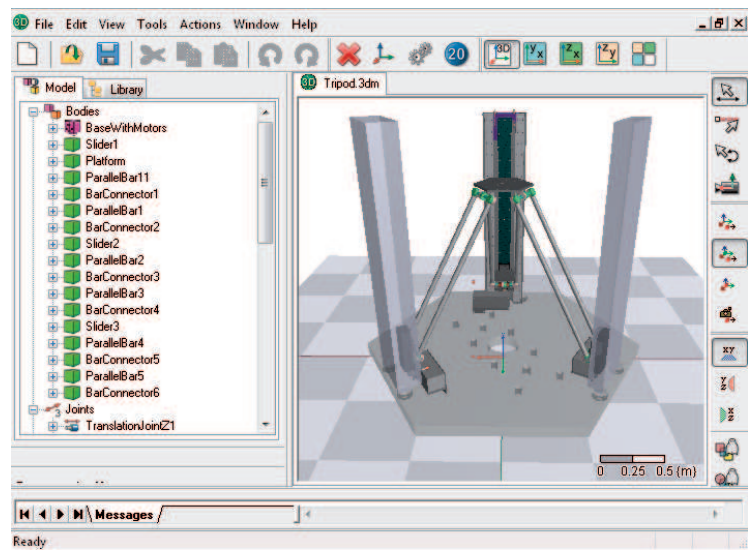

Fig. 12. Simulation of a Tripod with 20-sim 3D Mechanics Toolbox

\subsection{Multibody dynamic engines}

In the last years new simulation tools have been available based on the general engines for the simulation of physics environments (NGD, 2008; ODE, 1994; SD/FAST, 1994). These engines provide libraries for simulating the multi-body dynamics, i.e. the physics of motion of an assembly of constrained or restrained bodies. As such they encompass the behaviour of nearly every object and among them are, of course, also robot manipulators. These dynamic engines have beside the dynamics simulation engine also a collision detection engine. The collision engine is given information about the shape of each body and then it figures out which bodies touch each other and passes the resulting contact point information to the user. The user can then take the proper actions.

As an example we have selected the Open Dynamic Engine (ODE, 1994). Building the model of a robot is straightforward. First you have to create all bodies and connect them if desired with proper joints. For example, the 3DOF model as shown before can be defined as shown in Fig. 13. For comparison with MATLAB based tools the computational time for $n \mathrm{R}$ planar manipulators $(n=2, \ldots, 10)$ is shown in Fig. 10. It can be seen that the computational efficiency of ODE is comparable to the SD/FAST library.

Unfortunately, most of dynamics engines do not support functionality necessary to include robot models in the control algorithms. Advanced control algorithms including robot models include Jacobian matrices, inertia matrices, gravity forces, etc., and they are not explicitly defined. The user can use some implicit algorithms or other tools to get these parameters.

The dynamic simulation of multibody systems becomes very important when introducing robotics into human environments (Go et al., 2004; Khatib et al., 2002; Miller \& Christensen, 2003) where the success will not depend only on the capabilities of the real robots but also on the simulation of such systems. For example, in applications like virtual prototyping, teleoperation, training, collaborative work, and games, physical models are simulated and interacted with both human users and robots.

For example, the dynamics engine within a robotic grasping simulator known as GraspIt! (Miller \& Allen, 2004) computes the motions of a group of connected robot elements, such as an arm and a hand, under the influence of controlled motor forces, joint constraint forces, contact forces and external forces. This allows a user to dynamically simulate an entire grasping 


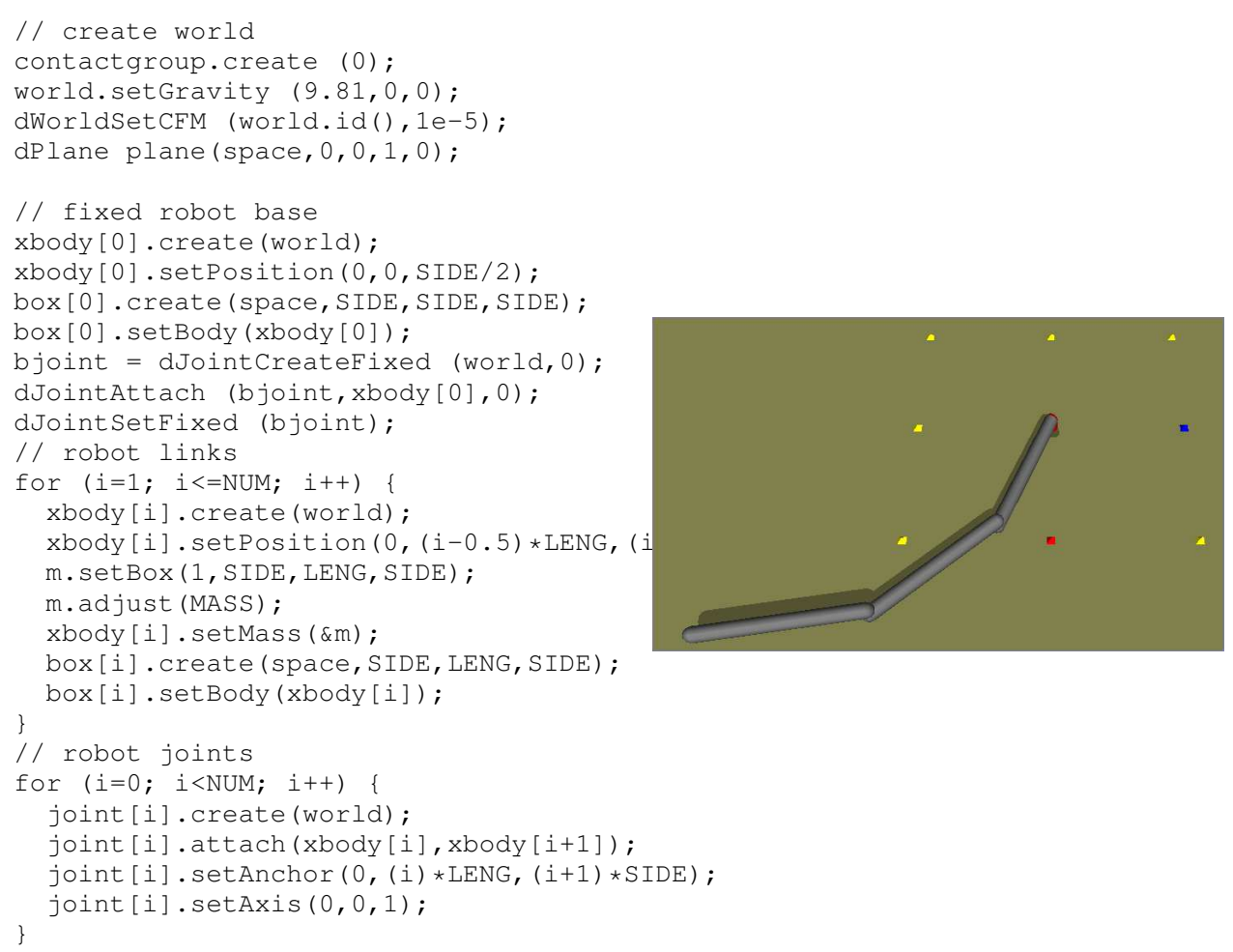

Fig. 13. Definition of 3R planar manipulator in ODE

task, as well as test custom robot control algorithms. Fig. 14 shows how a robot hand can grasp a mug (Miller \& Allen, 2004). In this example, all contacts between the fingers and the mug and related forces are analysed.

\section{Control design and integrated environment}

A very important part of the robotic system is the control system. In the process of controller design different steps have to be performed. First of all, the system has to be modelled. In the next step, the control algorithm is developed. The first results are then obtained by the simulation. If the results are satisfactory, then in the final stage the control algorithms are tested on a real system. For this, a real-time code should be generated and implemented on the real system. The integration of all these steps, although essential, is very difficult. Namely, the different steps in the development of the controller require the use of different methods for which different tools are needed. Hence, the results from one step to another have to be transferred often by hand. This bottleneck can be overcome if control design and testing are done in an integrated environment.

The importance of simulation tools in the development of robot control systems has been recognized by researchers very early. We have been using different simulation tools for over 20 years and many of them have been developed in our laboratory. In the last decade we have been using for the control design MATLAB/Simulink based integrated environment based 


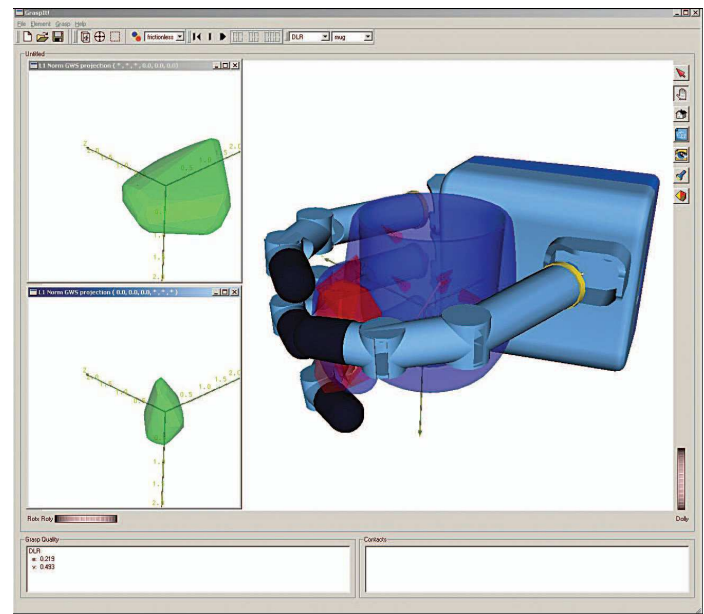

Fig. 14. A force-closure grasp of the mug using the DLR hand, which has a metal palm, inner link surfaces, and rubber fingertips.

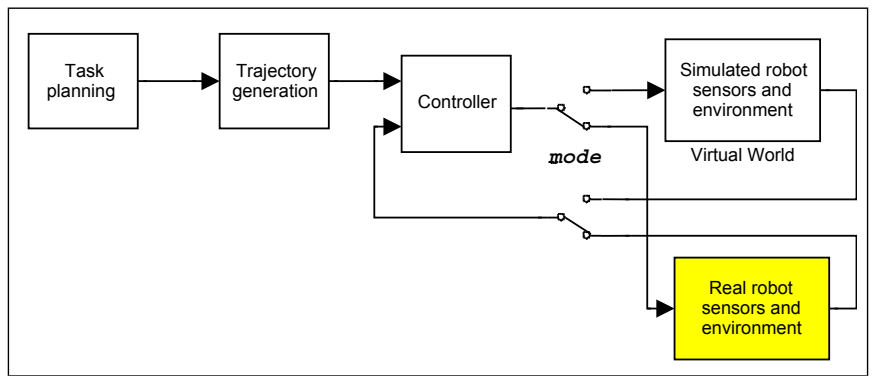

Fig. 15. A block diagram of the integrated environment

on Planar Manipulators Toolbox for dynamic simulation of redundant planar manipulators Žlajpah (2001). It enables the use of different sensors in the control loop and also the real-time implementation of the controller and hardware-in-the-loop simulation. Figure 15 shows the general simulation scheme in this environment. A crucial feature inherited in this scheme is indicated by the mode switches. Namely, the user can easy switch between using model or a real system in the simulation loop. This is one of the main features which we need for development of the robot control systems.

For example, Fig. 16 shows the dynamic model of a manipulator and a sensor detecting the object in the neighbourhood of the manipulator. When the developed controller is tested on a real system we substitute the manipulator with our experimental robot, i.e. the dashed blocks in Fig. 16 are replaced with the interface blocks as shown in Fig. 17a. Fig. 17b shows our laboratory manipulator with four revolute DOF acting in a plane, which has been developed specially for testing the different control algorithms for redundant robotic manipulators, performing an obstacle avoidance task.

The integration of the two modes is the most important feature of the integrated environment. This has been recognized also by many other researchers. For example, one of the goals of the 


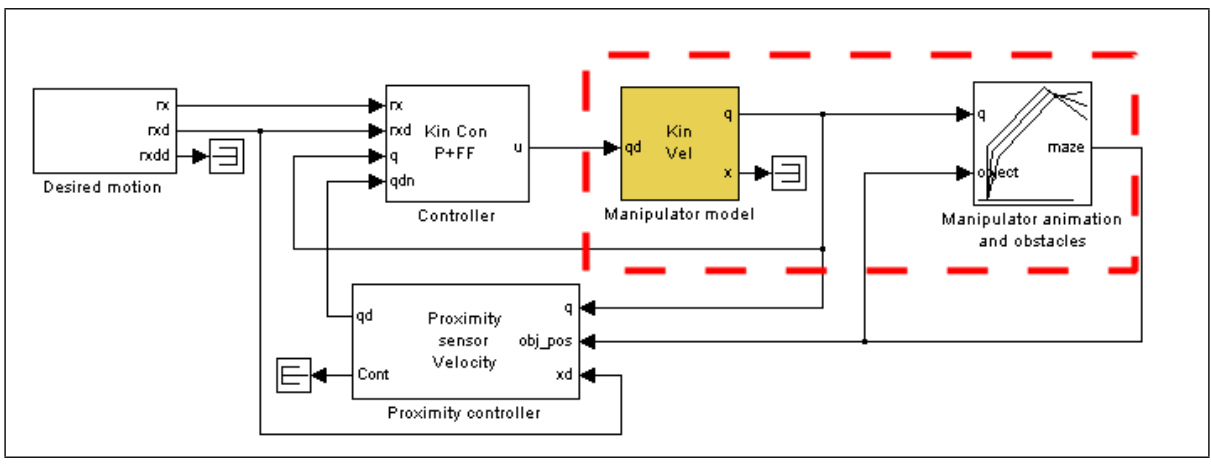

Fig. 16. A block diagram including the dynamic model of a manipulator and a sensor detecting the object in the neighbourhood of the manipulator

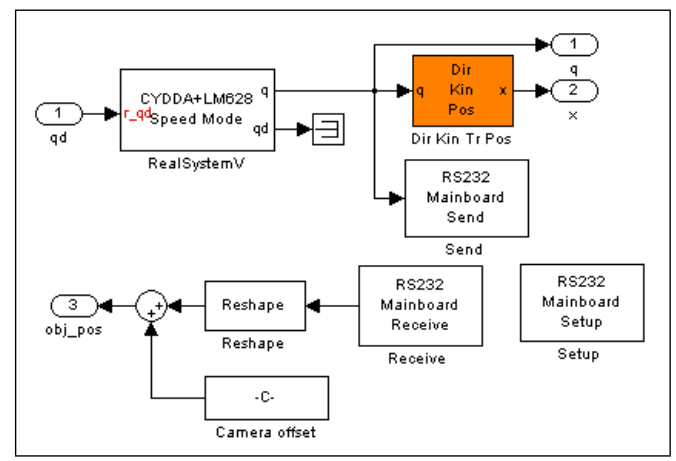

a) Robot interface block scheme

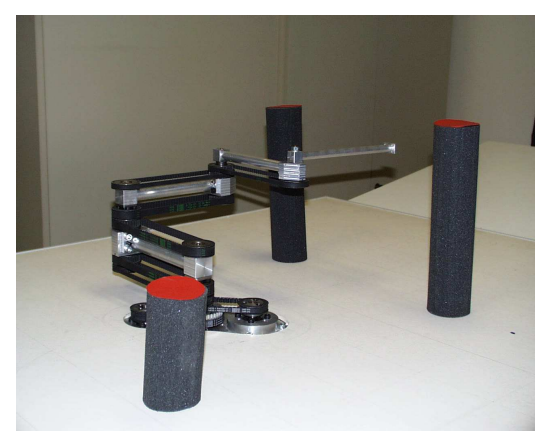

b) Experimental 4-R manipulator

Fig. 17. Avoiding obstacles - Hardware-in-the loop simulation 
IST project "RealSim" was to develop an efficient tool for modelling, simulating and optimising industrial robots (Kazi \& Merk, 2002). Fig. 18 shows the structure of the simulation system where a real control unit is connected to the simulator of an industrial robot. Using such system the controller can be tested without a real robot, e.g. before even the robot has been built.

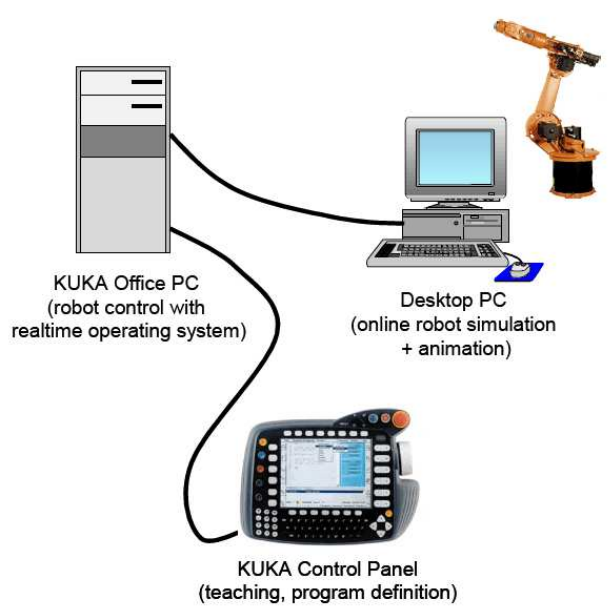

Fig. 18. Real time optimisation system (Project RealSim (Kazi \& Merk, 2002))

\subsection{The concept of distributed environment}

The Planar Manipulators Toolbox has proved to be a very useful and effective tool for many purposes, but it has been primary designed for the kinematic and dynamic simulation of the planar manipulators and to develop and test control algorithms on the lower control level, especially for redundant manipulators. In the last years, the scope of our research is oriented more in the development of control systems for humanoid and service robots (Gams et al., 2009; Omrčen et al., 2007). These robots have in general a more complex mechanical structure with many degrees-of-freedom. So, complex kinematic and dynamic models are necessary to simulate them. Furthermore, the control methods and algorithms are now usually a part of the higher robot control levels and the low level close-loop control algorithms are assumed to be a solved issue. These high level control algorithms can become very complex and may even require parallel computation distributed over more computers.

Considering all new requirements, which are:

to simulate the kinematics and dynamics of arbitrary chosen kinematic chain describing different manipulators,

to enable integration of different sensor systems like vision and force sensors,

to enable simulation of scenarios for complex robot tasks,

to include the model the robots' environments,

to visualize the robots and their environment and 


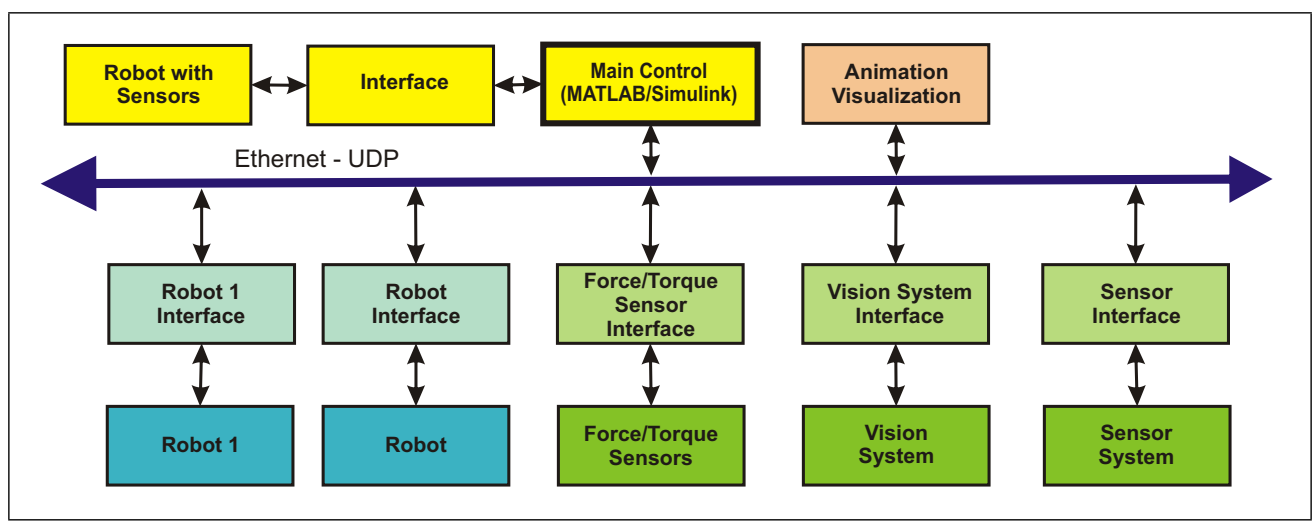

Fig. 19. A functional block diagram of the robot integrated environment in Robotics Laboratory including the robot PA10, mobile platform Nomad XR 4000 and sensor systems

to enable integration of real robots in the simulation loop, we have to reconsider the concept of the control design environment we will use in future. Based on our good experience with MATLAB/Simulink we have decided that this environment will be the kernel of our simulation tools. However, some of the above requirements can be easier fulfilled by using other tools. For example, the visualization of the robot and the environment can be easily done by dedicated graphics tools. Furthermore, advanced robot control strategies rely intensively on feedback sensor information. The most complex sensor system is the vision system, which can have several configurations and can be implemented on a single computer or on a computer cluster composed of many computers running different operating systems. To integrate such a diversity of hardware components in a unique framework we have decided to use the ethernet communication and the UDP protocol. In this way, we have maximal possible "degree-of-openness" of the system. Fig. 19 shows a typical scheme of our robot integrated environment.

In this scheme, each block can represent a real system or a model of that system. Note that because of using ethernet communication between the blocks, different software tools on different platforms can be used to simulate specific parts of the system. Consequently, the simulation environment can consist of several interacting applications, each representing a part of the system (Petrič et al., 2009).

\subsection{Simulink block library}

In Simulink, a system is modelled by combining input-output blocks. To gain the transparency, we try to represent a system by the block structure with several hierarchical levels, i.e. by combining different basic blocks, subsystems are built which become a single block at the higher level. In Figure 15 the typical robot subsystems can be seen: the trajectory generation, the controller, the model of the manipulator and the environment and the animation of manipulator motion. Figure 20 shows the Robot systems block library. The goal of the library is to provide blocks which are needed to simulate robotic systems and can not be modelled with standard blocks. First of all, these are the blocks for robot kinematic and dynamic models, the blocks for sensors systems, the typical transformations present in robot systems and the special interface blocks for robots, sensors and all other communications. Additionally, the 


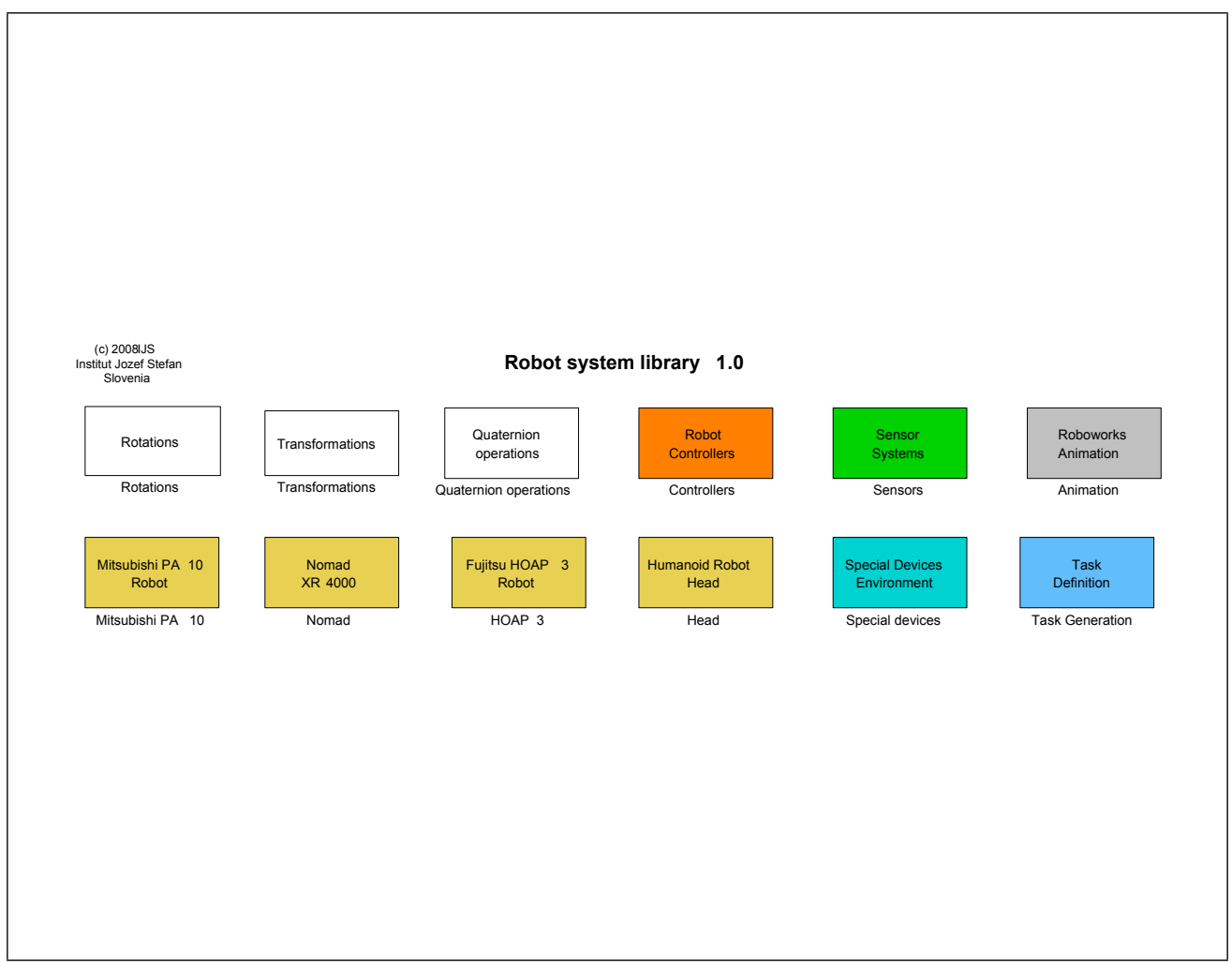

Fig. 20. Simulink Robot system library

library includes some blocks with standard subsystems like task space controllers, trajectory generation modules, etc.

\subsection{Integration of sensors}

Advanced robotics is characterized by the variety of complex sensory systems, e.g. vision sensors, force sensors, acoustic sensors, laser scanners, proximity sensor, etc. Therefore it is extremely important to apply as accurately as possible the sensor models into the simulation environment. The models of sensors are completely transparent to the design environment, i.e. real sensor can be substituted with the simulated one and vice versa in the control loop. The integration of sensors depends on their characteristics. Complex sensor systems like vision and acoustic sensors, or more advanced laser proximity sensors require relatively high computational power for signal processing. In many cases, it is difficult to accomplish all required data processing on the local computer. Often we have to apply a remote computer or even a remote computer cluster in order to obtain required computational power. In such a case, the subsystems are connected through ethernet with UDP protocol. We have developed a special protocol classes for different sensors, actuators and other subsystems. However, the performance is also affected by the communication delays. Therefore, it is favourable to pro- 


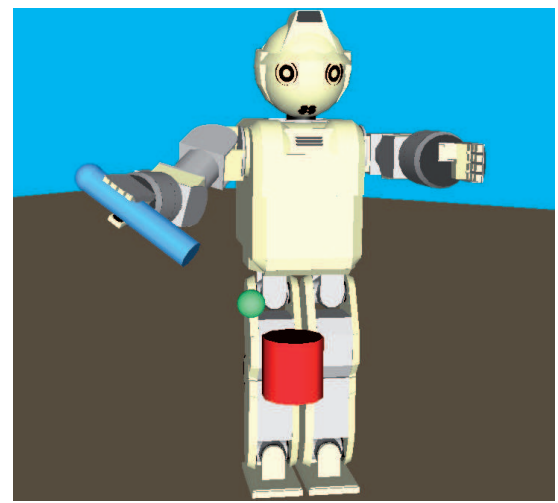

Fig. 21. Animation of the HOAP 3 humanoid robot using RoboWorks ${ }^{\mathrm{TM}}$

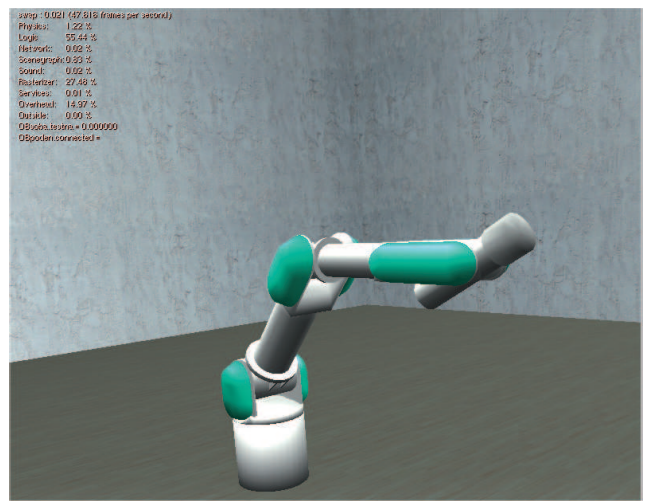

Fig. 22. Animation of the PA10 robot in Blender

cess signals of high frame-rate sensor, such as joint encoders, tachometers, force sensors, etc. on the local computer.

\subsection{Visualization and animation}

It is very important to visualize the simulation results. Especially in robotics it is necessary to "see" the motion of the robot and objects in the working environment. In our system we rely on external software for the visualization and animation of robots. In general, joint angles of robotic manipulators as well as the position and orientation of the other simulated objects in the scene are passed to the visualization tools using TCP/IP or UDP protocol. Currently, we have integrated into our simulation environment two visualization software packages RoboWorks (RoboWorks, 2008) and Blender(Blender, 2008).

Roboworks incorporates simple, but efficient modeler. Because of its simplicity the RoboWorks package is the favourable tool for the visualization of simpler systems, i.e. one or two robots in non-complex environment. Figure 21 shows the animation of our HOAP 3 humanoid robot and also in the following examples the RoboWorks environment has been used for the visualization.

For more complex scenes we use Blender, an open source multi-platform 3D computer animation program, which has a lot of features that are potentially interesting for engineering purposes, such as the simulation and programming of robots, machine tools, humans and animals, and the visualization and post-processing of all sorts of data that come out of such biological or artificial "devices". Blender supports also scripts (via Python interfaces to the core $\mathrm{C} / \mathrm{C}++$ code), hence it can be extended in many different ways. Among others, Blender has the capability of placing moving cameras at any link of the kinematic chain, it supports the real time photo realistic rendering for the virtual reality simulation and has also a physics engine for the simulation of the interactions between entities.

\subsection{Real-time simulation}

The real-time performance of the control algorithm is very important when dealing with lowlevel control. However, when developing higher level control algorithms, the real-time may be also important especially when high sample frequency improves the performance of the 
system. Therefore, when manipulator-in-the loop simulation is performed, the simulation system which controls the robot system has to provide the real-time capabilities and enable high sample frequencies. There are many real-time operating systems as Real Time Linux, QNX, EYRX, SMX, etc. Disadvantages of these operational systems are time-consuming software development and incompatibility with other systems. The algorithms are usually written in $\mathrm{C}$ or some other low-level programming language, where more sophisticated control algorithms require more time and increase the chance of error. Due to the above mentioned disadvantages of some real-time operation systems, we use the Matlab/Simulink and the xPC Target operation system whenever possible (Omrcen, 2007). xPC Target enables the real-time simulation and hardware-in-the-loop simulation using corresponding interfaces. It is a good prototyping tool that enables connecting Matlab/Simulink models to physical systems and executing simulation in real-time on PC-compatible hardware. As xPC Target supports also UDP communication, this was also one of the reasons to select the UDP for the communication between different applications in the simulation environment (Omrcen, 2007). Nevertheless, using Matlab/Simulink and xPC Target environment brings some disadvantages. Most of the hardware used for a robot control, which is available on the market, does not provide drivers for xPC Target. Therefore, we had to develop drivers for our our robots and sensors.

\subsection{Case study}

To show the efficiency, flexibility and usability of our control design environment we outline a typical experimental example using the Mitsubishi PA robot. The robot task is to play yo-yo, i.e. to keep the amplitude of the yo-yo at a desired level (Žlajpah, 2006). The yo-yo is tied to the tip of the robot. To be able to play the yo-yo it is necessary to know the position of the yo-yo and the force in the string or the velocity of the yo-yo (depending on the control algorithm). A WebCam has been used to measure the position of the yo-yo. To measure the string force a JR3 force/torque sensor mounted on the end-effector of the robot has been used. The experimental setup is shown in Fig. 23. The control should be implemented on PC's in MATLAB/SIMULINK environment and we wanted to use the PA10 motion control board which allows to control the end-effector positions of the robot.

In the first step of the control design when different control strategies have to be tested, we simulated the whole system in Simulink. We used the PA10 kinematic model and we had to develop a Simulink model of the yo-yo. The top level simulation scheme is shown in Figure

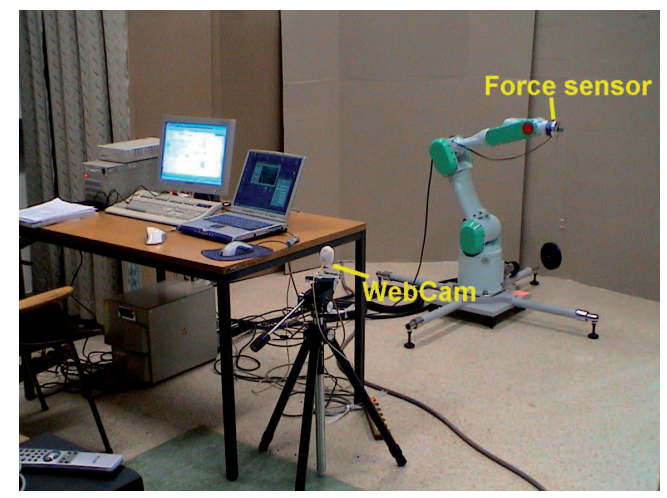

Fig. 23. Experimental setup (Mitsubishi PA10, vision system and force sensor) 

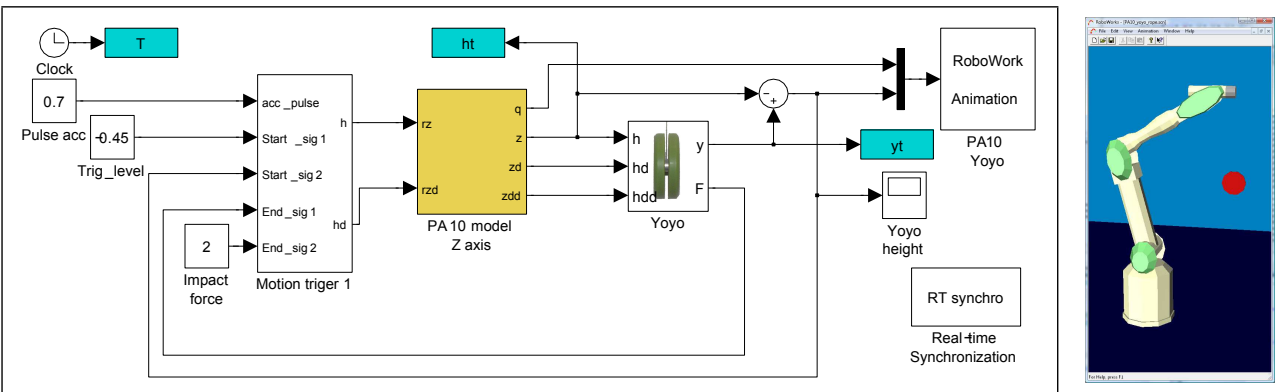

Fig. 24. Yoyo simulation: top level block scheme in Simulink and animation of the PA10 robot and yo-yo in RoboWorks

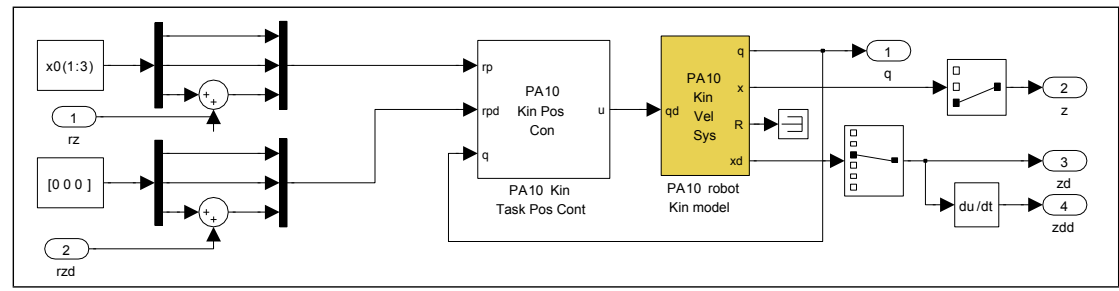

Fig. 25. PA10 model with kinematic task space position controller

24. The main three blocks are the controller, the robot model and a special model of the yo-yo (Žlajpah, 2006). As we want to move the robot end-effector only in the vertical direction the $z$-axis motion ( $x$ and $y$ positions are fixed to the initial values), we have to use a kinematic task space controller. This subsystem can be easily composed by combining blocks in our Simulink library as it is shown in Figure 25.

After the best control strategy has been verified using this simulation scheme, the next step is to test the control when the sensor systems information is obtained via ethernet connection. Therefore, we have developed a special yo-yo simulator, which receives the hand position and sends the position of the yo-yo and the string force via ethernet connection using UDP protocol (see Figure 28). The simulation scheme is the same except that instead of yo-yo Simulink model, the corresponding UDP interface blocks are used (see Figures 26 and 27).

As the external yo-yo simulator is a real time simulator, also in Simulink real-time simulation should be used. As the sampling frequency in this case is rather low $(100 \mathrm{~Hz}$ for robot control and $25 \mathrm{~Hz}$ for vision system) and the computation time of the Simulink model is small enough, we can use a special block for real-time synchronization.

Finally, when the designed control algorithms give satisfactory simulation results, we can test the control strategy on a real system. In manipulator-in-the-loop simulation, the model of the PA10 robot is replaced by the corresponding interface blocks. The position of the yo-yo and the string force are now obtained from the vision system and force sensor using the same interface as when the yo-yo simulator has been used. The corresponding scheme is shown in Figures 29. From the top level scheme it can easily be seen that the controller part of the system has not been changed and is the same as in the previous simulation schemes. 


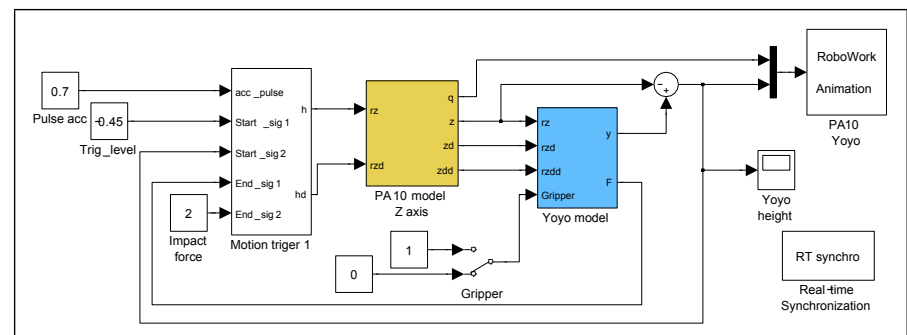

Fig. 26. The case with kinematic PA10 robot model and external yo-yo simulator

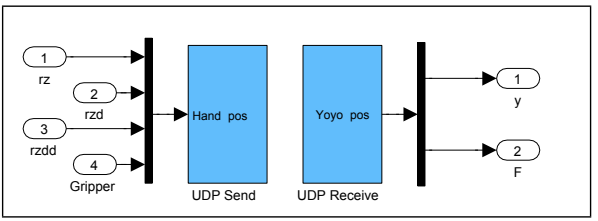

Fig. 27. Interface for external yo-yo simulator (Yoyo model block)

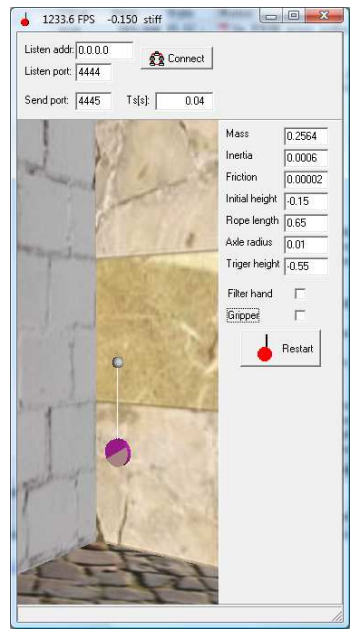

Fig. 28. External yo-yo simulator

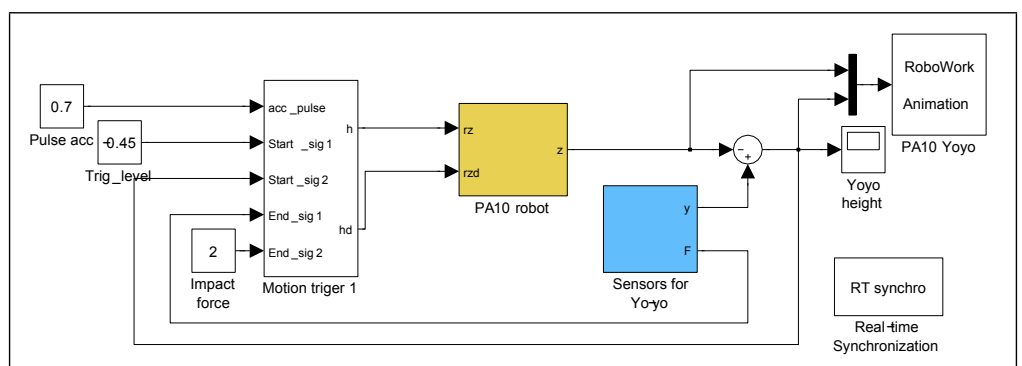

Fig. 29. Hardware-in-the-loop simulation (real PA10 robot, force sensor and vision systems are in the simulation loop)

\section{Overview of simulation in different robotic fields}

Robotics is very interdisciplinary, fast growing research field. Robots are introduced in various areas and used for different tasks also the requirements for the simulation tools depend on the particular application. However, all new applications, methodologies and technologies in robotics share the requirement to simulate robot systems and the environment with sufficient sophistication and accuracy. In the following, the role of the simulation tools in some robotic fields is presented.

\subsection{Off-line programming}

The greatest advantage of robots is their flexibility, i.e. their ability to be rearranged for new production tasks. Utilization of the robot's flexibility presupposes the effective programming. Robot can be programmed directly using the robot controller and other required equipment. However, to overcome the limitation that requires floor presence for programming and if we do not want that production equipment (robot and auxiliary devices) is not occupied during 


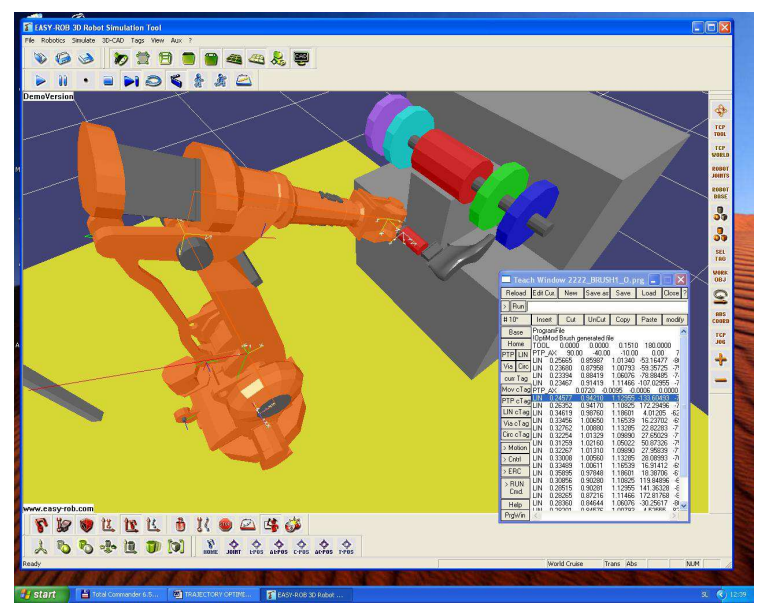

Fig. 30. Simulation of robot cell for the shoe finishing operations

programming the alternative is off-line programming. Off-line programming takes place on a separate computer, usually in the office and it uses the models of the workcell including robots and other devices that are used in the cell. Almost all commercially available industrial robots are supported by the off-line programming systems (ABB, 2008; Kuka, 2008; MotoSim, 2008). However, many other more general off-line programming systems are available for off-line programming and simulation of workcells which are not dedicated to a certain robot family (Easy-Rob, 2008; RobCAD, 1988; RobotWorks, 2008).

Using off-line programming systems robot programs can in most cases be created by the reuse of existing CAD data so that the programming will be quick and effective. Additionally, the robot programs are verified in simulation and any errors are corrected. For example, we have developed a robot cell for shoe finishing operation (creaming, brushing, etc.) where the trajectories have been generated using CAD data without considering the kinematic limitations of the robot and collisions with the objects in the workspace of the robot. Using off-line simulation these trajectories have been then verified and optimized before applied to the real robot (Fig. 30).

\subsection{Humanoid robots}

Advances in humanoid robotics open new possibilities of introducing humanoid robots into human environments. The goals of this emerging technology is that robots will work, assist, entertain and cooperate with humans or do certain jobs instead of a human. The foundation for these applications are control strategies and algorithms, sensory information and appropriate models of robot and environment. Virtual worlds, dynamic walking and haptic interaction are the topics addressed by researchers in this field (Hirukawa et al., 2003; Khatib et al., 2002; NASA, 2008; Stilman \& Kuffner, 2003).

As an example we show a simulation environment which allows the user to interactively control a virtual model of a humanoid (Stilman \& Kuffner, 2003). Fig. 31 shows a humanoid robot which is permitted to reconfigure the environment by moving obstacles and creating free space for a path. The software components include modelling of the robot geometry and 


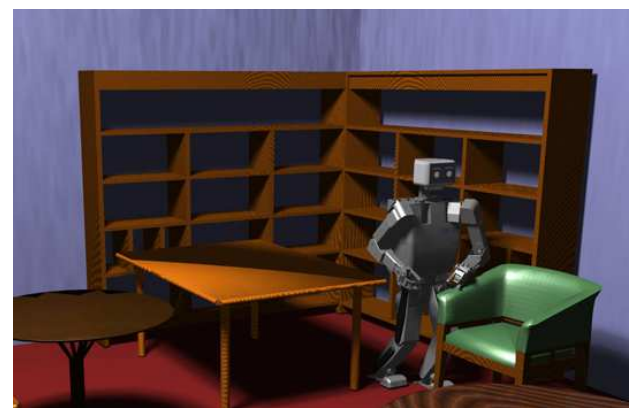

Fig. 31. Humanoid robot navigating among movable obstacles

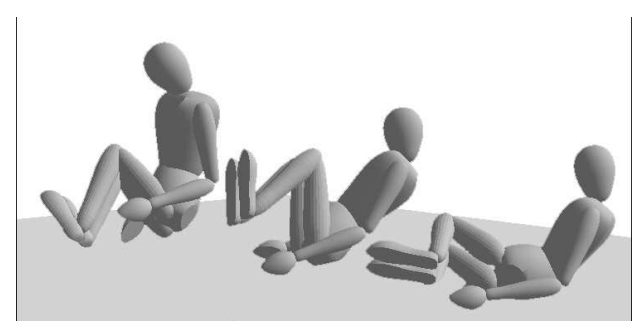

Fig. 32. Humanoid body falling due to gravity.

kinematics, simulated stereo visual sensing, and motion planning. In addition, the simulation environment serves as a graphical user interface for controlling the real robot hardware interactively.

The next example considers the whole-body behaviours (Khatib et al., 2005). Fig. 32 illustrates a virtual real-time simulation where a humanoid is falling under the effects of the gravity and colliding with the floor at multiple contact points. The proposed control was implemented and verified in a virtual environment that integrates multi-body dynamics, multi-robot control, multi-contact multi-body resolution and haptic interaction for robot teleoperation.

\subsection{Robotics in medicine}

The modelling of deformable organs, planning and simulation of robotics procedures, safe and real-time integration with augmented reality are the key topics in the field of robot assisted surgery (Ève et al., 2004). Fig. 33 shows the interface of the software designed at INRIA (Chir Robotics Medical Team) for the planning and simulation of robot assists surgical interventions. The simulator has the double aim of offering the surgeon a realistic environment to develop good control over the robot, and of validating the suggested incision ports.

\subsection{Mobile robotics}

Mobile robotics is a complex research area in which many advanced technologies and open research issues are combined all together. It is often difficult to master perfectly every technology, and hence realistic simulations and fast prototyping of mobile robots help to reduce the amount of time and hardware spent in developing mobile robotics applications. Moreover, the simulation tools allow the researchers to focus on the most interesting parts of their robotics projects and hence to achieve more advanced results. Especially, as mobile robots move out of laboratories and into the hands of users it is required that the simulation tools provide an integrated development environment for specifying, evaluating and deploying robot missions and it should allow non-expert users to specify robot missions in an easy way, e.g. using a visual programming paradigm. Furthermore, integrated support for evaluating solutions via simulation and finally deploying them on robots must also be available.

The Webots mobile robotics simulation software (Webots, 2005) provides you with a rapid prototyping environment for modelling, programming and simulating mobile robots. The included robot libraries enable you to transfer your control programs to many commercially available real mobile robots. Figure 34 shows the simulation of Sony AIBO robot. 


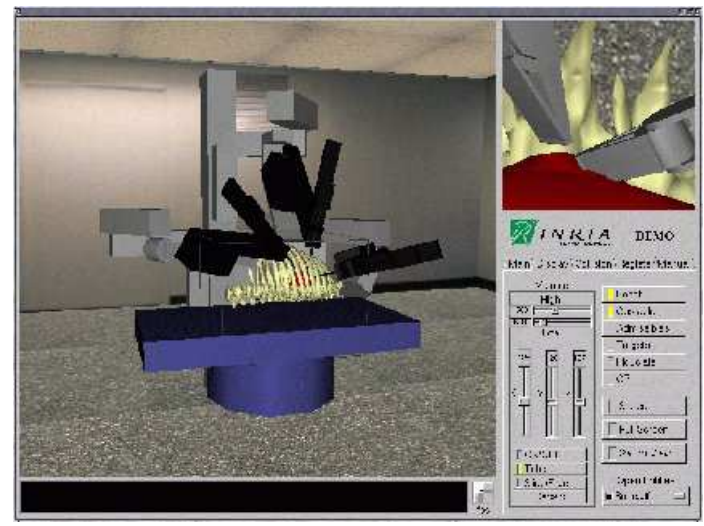

Fig. 33. Simulation of robot assisted surgical interventions (INRIA - ChIR)

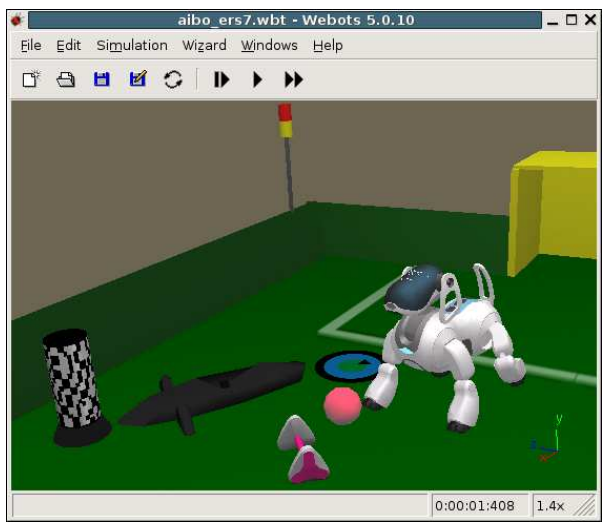

Fig. 34. Simulation of Sony Aibo ERS-7 in Webots simulator

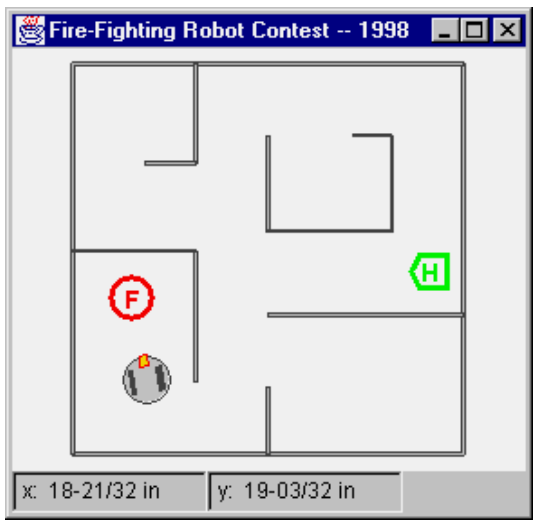

Fig. 35. Simulated competition arena from the Trinity College Fire Fighting Home Robot Contest 


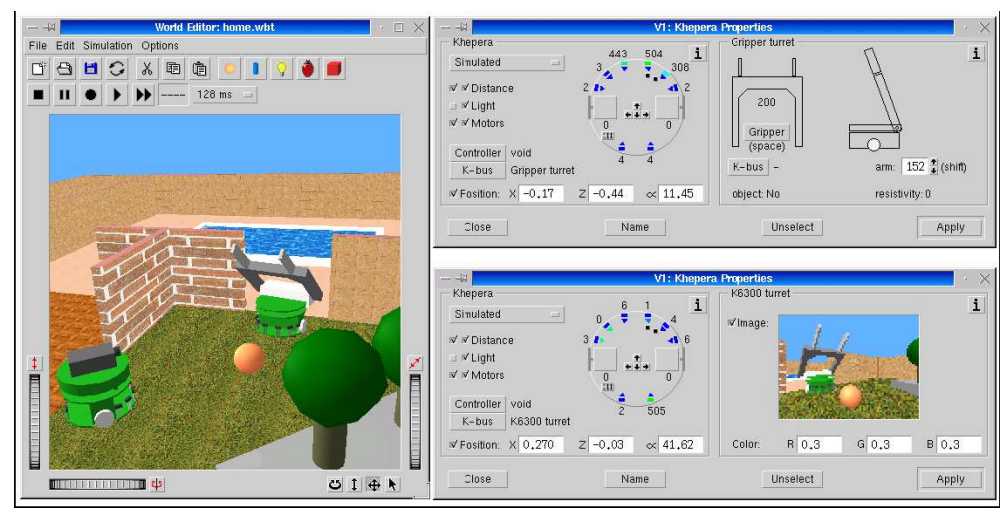

Fig. 36. Webots simulator: Simulation of Khepera robots

Rossum's Playhouse is a modest, two-dimensional robot simulation intended to aid developers implementing control and navigation logic. It allows applications to build a dataconfigurable robot which can interact with a simulated landscape or solve a virtual maze. It is primarily a tool for programmers and other developers who are writing the software for robotic applications. It can also be used as a testbed for algorithms and control logic. A number of developers are currently using the simulator to test and improve competition strategies for small robots. Fig. 35 shows a maze-solving application in a known environment.

Some of the simulation programs are designed for special robots. A very popular mobile robot used in research is Khepera and there are many simulation packages which support this robot, like Webots (Michel, 2008) (Fig. 36) or Matlab based toolbox KiKS (Storm, 2008). Another example is the EyeSim simulator (EyeSim, 2008). EyeSim is a multiple mobile robot simulator that allows experimenting with EyeBot mobile robots. The user can test the same unchanged programs that run on the real robots. In Fig. 37 a 3D scene representation of the environment and robots in it is being shown, together with the views of active robots.

A special kind of mobile robots are biomimetic robots which borrow their structure and senses from animals, such as insects. The most well-known early biomimetic robots were a cockroach and a lobster. The research is aimed at developing new mobile robots that exhibit much greater robustness in performance in unstructured environments than mobile robots with wheels. These new robots will be substantially more compliant and stable than current robots, and will take advantage of new developments in materials, fabrication technologies, sensors and actuators. Applications will include autonomous or semi-autonomous tasks in an obstaclestrewn ground or a sloshy ocean bay. Also here simulation plays an important role.

Fig. 38 shows a walking bug (Reichler \& Delcomyn, 2000). The simulation system is able to simulate anything from a single leg segment to an entire walking insect, including muscles, sense organs, and the nervous control system. The user can enter the physical dimensions of a single leg of an insect, place muscles and sense organs where they are known to be located on the leg of a living insect, and study how the nervous system might use these components to generate reflex movements produced by a stimulus applied to one of the sense organs. By entering the physical dimensions of the entire body as well as all six legs, it is possible to study the way in which the nervous system might generate coordinated walking under various 


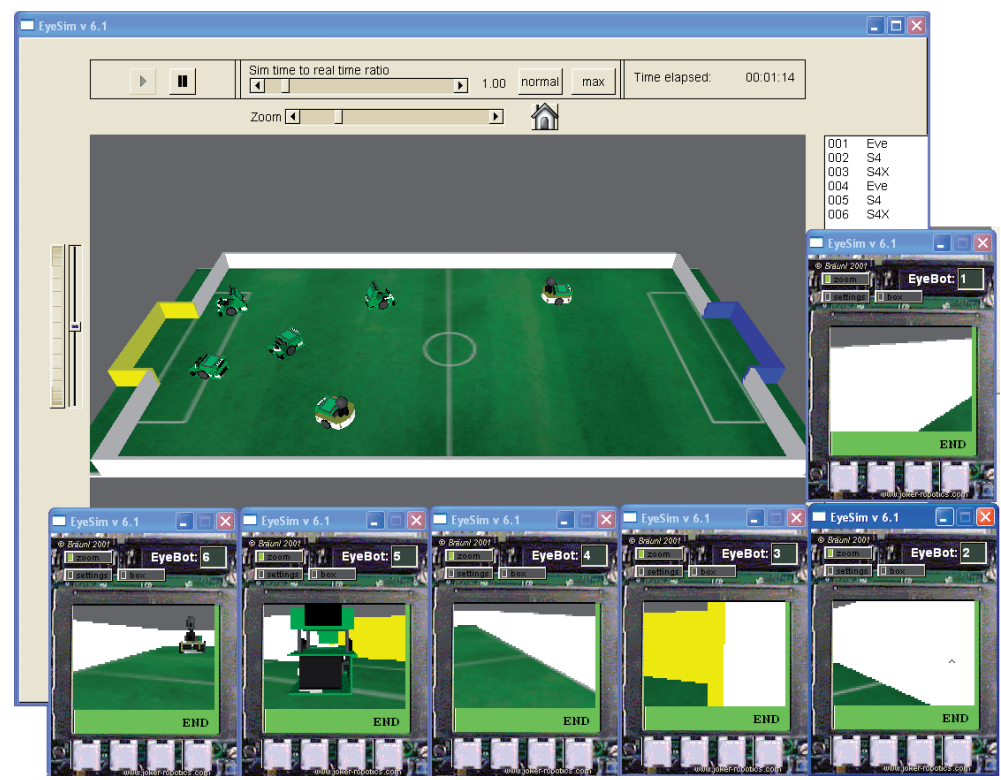

Fig. 37. EyeSim simulator: EyeBots playing football

circumstances. The nervous system can also be emulated flexibly - as a single "controller" or as a hierarchical chain of controllers, as desired.

\subsection{Nanorobotics}

The automation, control, and manufacturing of nanorobots is a challenging and very new field. The design and the development of complex nanomechatronic systems with high performance should be addressed via simulation to help pave the way for future applications of nanorobots in biomedical engineering problems. Successful nanorobotic systems must be able to respond efficiently in real time to change the aspects of microenvironments. Hence, simulation tools should not provide only animation or visualization, but should encounter also physical characteristics of nanorobots and environment (Cavalcanti \& Jr., 2005). Fig. 39 shows the virtual environment which is inhabited by nanorobots, biomolecules, obstacles, and organ inlets.

\subsection{Space robotics}

The behaviour of free-floating manipulators in space is different compared to the manipulators "on ground" because they are not fixed, the gravity is negligible and due to the conservation of linear and angular momentum. This makes control and planning of robotic end effector trajectories highly complicated (Dubowsky \& Papadopoulos, 1993). The simulation plays an important role in complex trajectory planning task especially as the space manipulator can have more than conventional number of six degrees of freedom. Additionally, the robotics simulation tools for space applications are designed also to meet the challenges of astronaut and ground personnel training a well as providing a valuable tool for space operations support. The simulator supports the critical tasks to be performed by astronauts, 


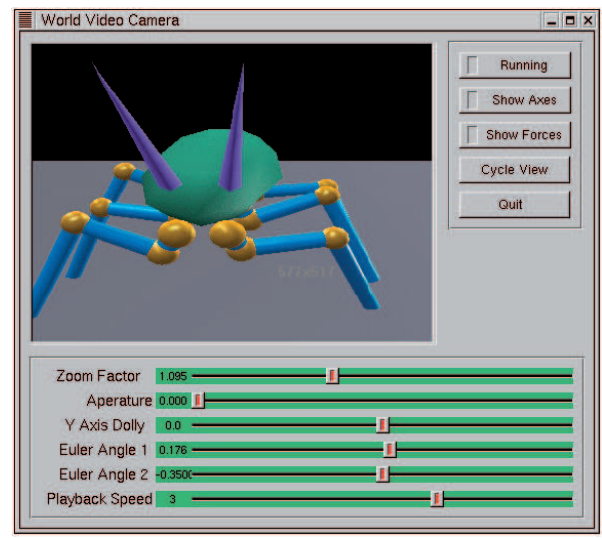

Fig. 38. A simulation of a walking insect

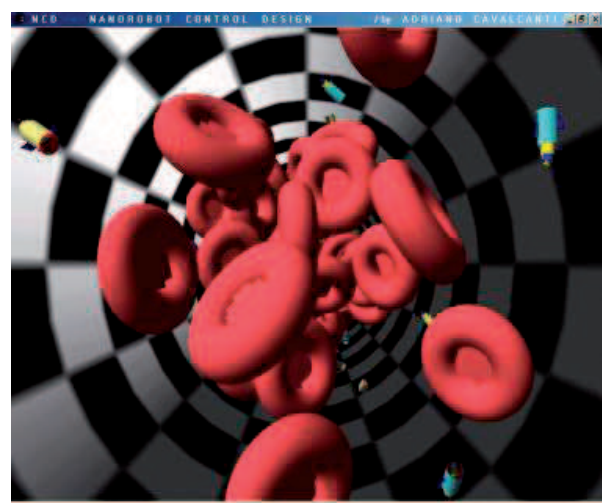

Fig. 39. View of simulator workspace showing the vessel wall with a grid texture, cells and nanorobots

including payload handling, berthing and de-berthing. For that it has to provide real-time high-fidelity simulation of the flexible dynamics performance of robotic arms and if necessary also of astronauts, contact dynamics models, and 3D visual models have to support realistic views generated by cameras in an operational and dynamic lighting environment that includes the production of split screen views. Fig. 40 shows a simulation of a humanoid robot Robonaut in space. The Robonaut simulation has been developed to bridge a gap between operations and development activities (NASA, 2008). The simulator matches the appearance, kinematics and dynamics of Robonaut and serves as a platform to test new control theories and configurations without having to use the real Robonaut or to construct new expensive hardware. Another benefit of the simulation regards path planning. Now operators can test arm motions on difficult tasks before actual operation of the robot, thus minimizing the risk to the hardware. 


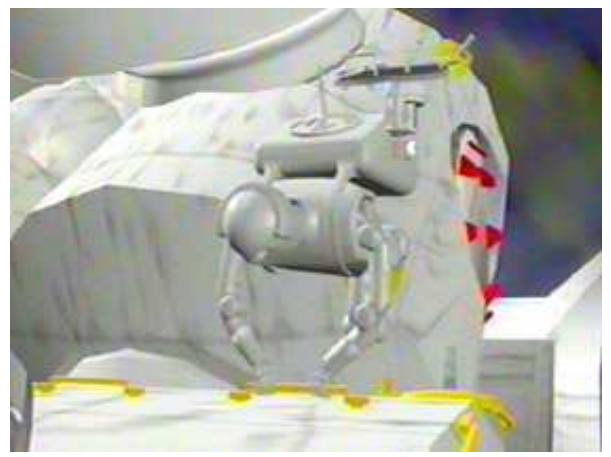

Fig. 40. The Robonaut simulator

\section{Conclusion}

The simulation is widely used in all fields of robotics from kinematics and dynamics to industrial applications. Actually, advanced robot systems require sophisticated simulation tools which can model accurately enough the physical world at a sufficient speed and allow the user interaction. New challenges in the simulation of the robotic systems are multi-body dynamics that computes robot and object motions under the influence of external forces, fast collision detection and contact determination, realistic visualization of the robot and environment, and haptic interaction. The advanced simulation tools are the foundation for the design of sophisticated robot systems, for the application of robots in complex environments and for the development of new control strategies and algorithms. The simulation being once a tool for the analysis of a robot system and task planning, has become an open platform for developing new robot systems. Not only that the modern simulation tools can simulate and visualize the real world in a very realistic way, they allow to go beyond the reality. Namely, the researchers may build experimental environments according to their own imagination, using robots and technologies which are not available yet. In the end, we believe that the simulation in robotics has reached a very important role and by using different simulation software, the current and future capabilities of complex robotic systems can be significantly improved.

\section{References}

ABB (2008). RobotStudio 5, ABB, http://www.robotstudio.com/rs5/.

Alotto, G., Bona, B. \& Calvelli, T. (2004). Prototyping Advanced Real-Time Robotic Controllers on Linux RTAI Systems with Automatic Code Generation, Proceedings of International Conference Mechatronics and Robotics 2004, Aachen, Germany.

Blender (2008). Blender: http://www.blender.org/.

Cavalcanti, A. \& Jr., R. A. F. (2005). Nanorobotics Control Design: A Collective Behavior Approach for Medicine, IEEE Transactions on NanoBioScience (2): 133 - 140.

Corke, P. I. (1996). A Robotics Toolbox for MATLAB, IEEE Robotics \& Automation Magazine 3(1): $24-32$.

Dubowsky, S. \& Papadopoulos, E. (1993). The Kinematics, Dynamics, and Control of FreeFlying and Free-Floating Space Robotic Systems, IEEE Trans. on Robotics and Automation, Special Issue on Space Robotics 9(5): 531 - 543.

Easy-Rob (2008). Easy-Rob, 3D Robot Simulation Tool, http:/ /www.easy-rob.de/. 
Ève, C.-M., Adhami, L., Mourgues, F. \& Bantiche, O. (2004). Optimal planning of robotically assisted heart surgery: Transfer precision in the operating room, International Journal of Robotics Research (4): 539-548.

EyeSim (2008). EyeSim, EyeBot Simulator, http:/ / robotics.ee.uwa.edu.au/eyebot/index.html.

Fenton, R. \& Xi, F. (1994). Computational analysis of robot kinematics, dynamics, and controlusing the algebra of rotations, IEEE Trans. on Systems, Man, Cybernetics (6): 936 942.

Gams, A., Ijspeert, A. J., Schaal, S. \& Lenarčič, J. (2009). On-line learning and modulation of periodic movements with nonlinear dynamical systems, Autonomous Robots 27(1): 3 $-23$.

Go, J., Browning, B. \& Veloso, M. (2004). Accurate and flexible simulation for dynamic, visioncentric robots, Proceedings of International Joint Conference on Autonomous Agents and Multi-Agent Systems (AAMAS'04), New York.

Hirukawa, H., Kanehiro, F., Kajita, S., Fujiwara, K., Yokoi, K., Kaneko, K. \& Harada, K. (2003). Experimental Evaluation of the Dynamic Simulation of Biped Walking of Humanoid Robots, Proc. IEEE Int. Conf. on Robotics and Automation, Taipei, Taiwan, pp. $1640-$ 1645 .

Kazi, A. \& Merk, G. (2002). Experience with RealSim for Robot Applications, Technical report, KUKA Roboter $\mathrm{GmbH}$, Augsburg.

Khatib, O., Brock, O., Chang, K.-S., Conti, F. \& Ruspini, D. (2002). Human-Centered Robotics and Interactive Haptic Simulation, Int. J. of Robotic Research 23(2): 167 - 178.

Khatib, O., Sentis, L., Park, J.-H. \& Warren, J. (2005). Whole Body Dynamic Behavior and Control of Human-Like Robots, International Journal of Humanoid Robotics (1): 29-43.

Kleijn, C. (2009). Getting Started with 20-sim 4.1, Controllab Products B.V.

Kuka (2008). KUKA.Sim, KUKA Industrial robots, http://www.kukarobotics.com/en/products/software/kuka_sim/.

Žlajpah, L. (2006). Robotic yo-yo : modelling and control strategies, Robotica 24(2): 211 - 220.

Lambert, J. M., Moore, B. \& Ahmadi, M. (2001). Essential Real-Time and Modeling tools for Robot Rapid Prototyping, Proceeding of the 6 th International Symposium on Artificial Intelligence and Robotics \& Automation in Space i-SAIRAS 2001, Quebec, Canada.

Latombe, J.-C. (1995). Controllability, recognizability, and complexity issues in robot motion planning, Proc. of the 36th Annual Symposium on Foundations of Computer Science, Los Alamitos, CA, USA, pp. $484-500$.

Lippiello, V., Villani, L. \& Siciliano, B. (2007). An open architecture for sensory feedback control of a dual-arm industrial robotic cell, An International Journal Industrial Robot 34(1): 46-53.

MCA2 (2008). Modular Controller Architecture 2 - MCA2: http:/ /mca2.org/.

Michel, O. (2008). Webots: A Powerful Simulator for the Khepera Robot, http://www.ccnacht.de/_ccnacht2/media/pdf/khepera_webots.pdf.

Miller, A. \& Allen, P. K. (2004). Graspit!: A versatile simulator for robotic grasping, IEEE Robotics and Automation Magazine 11(4): 110 - 122.

Miller, A. T. \& Christensen, H. I. (2003). Implementation of multi-rigid-body dynamics within a robotic grasping simulator, Proc. IEEE Int. Conf. on Robotics and Automation, Taipei, Taiwan, pp. $2262-2268$.

MotoSim (2008). Solutions in Motion - MotoSim, Motoman Yaskawa Company, http:/ / www.motoman.com. 
MSRS (2008). Microsoft Robotics Studio. http://msdn2.microsoft.com/enus/robotics/default.aspx.

NASA (2008). Robonaut, NASA, http:/ / vesuvius.jsc.nasa.gov/er_er/html/robonaut/robonaut.html.

Nethery, J. \& Spong, M. (1994). Robotica: a Mathematica package for robot analysis, IEEE Robotics $\mathcal{E}$ Automation Magazine 1(1).

NGD (2008). Newton Game Dynamics, http://physicsengine.com/.

ODE (1994). Open Dynamics Engine, http:/ / ode.org/ode.html.

Omrcen, D. (2007). Developing matlab simulink and xpc target real-time control environment for humanoid jumping robot, 16th Int. Workshop on Robotics in Alpe-Adria-Danube Region - RAAD 2007, Ljubljana, Slovenia, pp. 18-23.

Omrčen, D., Žlajpah, L. \& Nemec, B. (2007). Compensation of velocity and/or acceleration joint saturation applied to redundant manipulator, Robot. auton. syst.. 55(4): 337 - 344.

Petrič, T., Gams, A. \& Žlajpah, L. (2009). Controlling yo-yo and gyroscopic device with nonlinear dynamic systems, Proceedings of $18^{\text {th }}$ Int. Workshop on Robotics in Alpe-AdriaDanube Region, Brasov, Romania, p. 6.

Reichler, J. A. \& Delcomyn, F. (2000). Dynamics Simulation and Controller Interfacing for Legged Robots, Int. J. of Robotic Research (1): 41 - 57.

RobCAD (1988). ROBCAD/Workcell, User's manual, Tecnomatix.

RobotWorks (2008). RobotWorks - a Robotics Interface and Trajectory generator for SolidWorks, http:/ /www.robotworks-eu.com/.

RoboWorks (2008). RoboWorks ${ }^{\mathrm{TM}}$ : http:/ / www.newtonium.com/public_html/Products/RoboWorks / RoboWorks.htm.

SD/FAST (1994). SD/FAST User's Manual, Symblic Dynamics, Inc.

SimMechanics (2005). SimMechanics, User's Guide, The Mathworks.

Stilman, M. \& Kuffner, J. (2003). Navigation Among Movable Obstacles: Real-time Reasoning in Complex Environments, Proc. IEEE Int. Conf. on Humanoid Robotics, Los Angeles, CA, USA.

Storm, T. (2008). KiKS is a Khepera Simulator, user guide, http://www.tstorm.se/projects/kiks/.

Žlajpah, L. (1997). Planar Manipulators Toolbox: User's Guide, Technical Report DP - 7791, Jožef Stefan Institute. URL: http://www2.ijs.si/ leon/planman.html.

Žlajpah, L. (2001). Integrated environment for modelling, simulation and control design for robotic manipulators, Journal of Intelligent and Robotic Systems 32(2): 219 - 234.

Webots (2005). Webots User Guide, Cyberbotics Ltd.

Zhang, Y. \& Paul, R. P. (1988). Robot Manipulator Control and Computational Cost, Technical Report MS-CIS-88-10, University of Pennsylvania Department of Computer and Information Science. http://repository.upenn.edu/cis reports/621. 


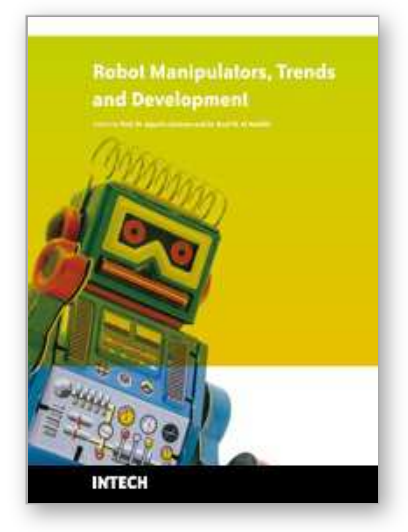

\author{
Robot Manipulators Trends and Development \\ Edited by Agustin Jimenez and Basil M Al Hadithi
}

ISBN 978-953-307-073-5

Hard cover, 666 pages

Publisher InTech

Published online 01, March, 2010

Published in print edition March, 2010

This book presents the most recent research advances in robot manipulators. It offers a complete survey to the kinematic and dynamic modelling, simulation, computer vision, software engineering, optimization and design of control algorithms applied for robotic systems. It is devoted for a large scale of applications, such as manufacturing, manipulation, medicine and automation. Several control methods are included such as optimal, adaptive, robust, force, fuzzy and neural network control strategies. The trajectory planning is discussed in details for point-to-point and path motions control. The results in obtained in this book are expected to be of great interest for researchers, engineers, scientists and students, in engineering studies and industrial sectors related to robot modelling, design, control, and application. The book also details theoretical, mathematical and practical requirements for mathematicians and control engineers. It surveys recent techniques in modelling, computer simulation and implementation of advanced and intelligent controllers.

\title{
How to reference
}

In order to correctly reference this scholarly work, feel free to copy and paste the following:

Leon Zlajpah (2010). Robot Simulation for Control Design, Robot Manipulators Trends and Development, Agustin Jimenez and Basil M Al Hadithi (Ed.), ISBN: 978-953-307-073-5, InTech, Available from: http://www.intechopen.com/books/robot-manipulators-trends-and-development/robot-simulation-for-controldesign

\section{INTECH}

open science | open minds

\author{
InTech Europe \\ University Campus STeP Ri \\ Slavka Krautzeka 83/A \\ 51000 Rijeka, Croatia \\ Phone: +385 (51) 770447 \\ Fax: +385 (51) 686166 \\ www.intechopen.com
}

\author{
InTech China \\ Unit 405, Office Block, Hotel Equatorial Shanghai \\ No.65, Yan An Road (West), Shanghai, 200040, China \\ 中国上海市延安西路65号上海国际贵都大饭店办公楼 405 单元 \\ Phone: +86-21-62489820 \\ Fax: $+86-21-62489821$
}


(C) 2010 The Author(s). Licensee IntechOpen. This chapter is distributed under the terms of the Creative Commons Attribution-NonCommercialShareAlike-3.0 License, which permits use, distribution and reproduction for non-commercial purposes, provided the original is properly cited and derivative works building on this content are distributed under the same license. 